Nouvelles perspectives en sciences sociales

\title{
Complexité de l'objet et catégories analytiques. Analyse d'un corpus sur divers organismes internationaux
}

\section{Roger Gervais et Simon Laflamme}

Volume 4, numéro 2, avril 2009

Sur le thème de la complexité

URI : https://id.erudit.org/iderudit/029894ar

DOI : https://doi.org/10.7202/029894ar

Aller au sommaire du numéro

Éditeur(s)

Prise de parole

ISSN

1712-8307 (imprimé)

1918-7475 (numérique)

Découvrir la revue

Citer cet article

Gervais, R. \& Laflamme, S. (2009). Complexité de l'objet et catégories analytiques. Analyse d'un corpus sur divers organismes internationaux. Nouvelles perspectives en sciences sociales, 4(2), 85-114.

https://doi.org/10.7202/029894ar
Résumé de l'article

Cet article vérifie trois hypothèses d'ordre épistémologique. La première veut que les sciences humaines, quand elles se penchent sur une question, le font presque par nécessité dans un cadre interdisciplinaire. La seconde propose que l'ensemble des écrits des chercheurs des sciences humaines, quand il porte sur une question particulière, peut être ramené, dans ses grandes lignes, à un nombre limité de catégories sémantiques. La troisième hypothèse suggère que l'analytique et l'éthique soient fortement associés. Pour vérifier ces hypothèses, l'analyse porte sur un corpus de milliers de résumés d'articles qui ont été publiés dans des revues avec comité de lecture et qui ont pour objet les organismes internationaux. Les trois hypothèses trouvent confirmation. 


\title{
Complexité de l'objet et catégories analytiques. \\ Analyse d'un corpus sur divers organismes internationaux
}

\author{
Roger Gervais \\ CIRESS-LEREPS-GRES, \\ Université de Toulouse 1/Université Laurentienne, Sudbury
}

Simon LAFLAmme Université Laurentienne, Sudbury

\section{Les sciences humaines et leur objet}

Tes objets des sciences humaines sont presque par essence Linterdisciplinaires. Cela est en grande partie attribuable au caractère composite des phénomènes qu' elles perçoivent. Comment, en effet, ces phénomènes pourraient-ils se réduire au seul aspect qu'une spécialisation examine quand elle subdivise en ses parties la réalité? C'est largement parce que, concrètement, les objets qui se donnent aux sciences sont indivisibles que la philosophie s'est souvent donné pour mission de synthétiser les connaissances ${ }^{1}$. C'est aussi pour cette raison que des approches généralement

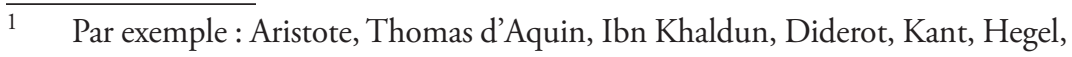
Marx, Morin. 
construites dans une logique systémique ${ }^{2}$ ou structurale ${ }^{3}$ se sont imposé d'aborder leurs objets en en reliant les éléments décomposés par la monodisciplinarité et par la spécialisation subdivisante. Mais le généralisme de la philosophie et l'obligation de réunir les parties des objets ou les objets eux-mêmes les uns par rapport aux autres sont loin d'être dominants dans les sciences humaines. La monodisciplinarité constitue le milieu à l'intérieur duquel sont fabriquées le plus communément les connaissances - ce qui soulève, d'ailleurs, des réactions exaspérées ${ }^{4}$. Mais on peut se demander si ce spécialisme parvient réellement à isoler les composantes du social et de l'humain. Il nous semble en effet que, si tournées vers la spécialisation que soient les sciences humaines, quand on aborde l'ensemble des recherches qu'elles produisent, elles font état du caractère indissociable des composants de l'humain et de la société. Non seulement en est-il ainsi, croyons-nous, mais il nous apparaît de surcroît que l'ensemble des catégories à l'intérieur desquelles les chercheurs peuvent aborder une question est relativement limité et que cette communalité témoigne d'une espèce d'entente tacite sur ce qui constitue les aspects les plus fondamentaux de la socialité. Combien de penseurs de la société ne voient pas dans l'économie une dimension incontournable de la vie humaine? Combien de chercheurs n’ont pas invoqué la nécessité de combiner dans une

2 Par exemple : Yves Barel (Le paradoxe et le système, Grenoble, Presses universitaires de Grenoble, 1989), Ludwig von Bertalanffy (General System Theory: Foundations, Development, Applications, New York, George Braziller, [1968] 1976), Niklas Luhmann (Social Systems, Stanford (CA), Stanford University Press, [1984] 1995), Talcott Parsons (The Social System, Glencoe (Ill.), Free Press, 1951).

3 Par exemple : Pierre Bourdieu (La reproduction. Elément pour une théorie du système d'enseignement, Paris, Minuit, 1970), Claude Lévi-Strauss (Les structures élémentaires de la parenté, Paris, Mouton, Maison des sciences de l'homme, coll. " de Rééditions ", [1967] 1973), Antony Giddens (The Constitution of Society: Outline of the Theory of Structuration, Berkely (CA), University of California Press, 1984).

4 Par exemple : José Ortega y Gasset ("La barbarie du "spécialisme” ", dans La révolte des masses, Paris, Gallimard, coll. "Idées ", [1930] 1961), Basarab Nicolescu (La transdisciplinarité. Manifeste, Éditions du Rocher, coll. "Transdisciplinarité », 1996). 
analyse de la société le politique et l'économique ? Mentionnons les Karl Marx qui, même s'ils accordent quelque antécédent à l'économie, établissent une dialectique entre elle et le politique et, plus généralement, l'idéologique ; signalons les Lévi-Strauss qui se préoccupent de la circulation des biens, des idées et des personnes $^{6}$. On peut lire cette citation d'Edgar Morin :

Comme nous le montre notre histoire passée, toute idée de nature a fortement rétroagi comme mythe culturel, voire social et politique, sur la société où elle prenait corps. Aujourd'hui, dès sa naissance, l'éconature a rétroagi, non seulement sur nos idées et croyances, mais aussi sur des processus économiques, sociaux et politiques ${ }^{7}$;

ou celle-ci de Philippe Moreau Defarges :

Quatre types de flux créent, font vivre la mondialisation : ils concernent les hommes, les biens et les services, l'argent, enfin, les informations, les idées, les représentations mentales ${ }^{8}$.

Il nous semble donc que si l'on se penchait sur l'ensemble des travaux que les chercheurs ont publiés dans un secteur donné, on devrait trouver la trace aussi bien de l'indivisibilité du social que de la récurrence de catégories fondamentales.

Les sciences humaines sont bien équipées pour objectiver ce qu'elles étudient. Elles disposent en effet de méthodes et de théories qui permettent au chercheur de se désubjectiver, c'està-dire de faire que son discours ne soit pas réductible à sa subjectivité ou à ses orientations idéologiques. De même, elles sont soumises à des contraintes de vérification des propositions qu'elles avancent, de logique des discours qu'elles construisent et de communication des résultats qu'elles obtiennent. Tout cela contribue à favoriser la scientificité de leur propos. Malgré cela, il nous semble que les spécialistes des sciences humaines ont à ce point de la difficulté à abandonner les considérations morales que, dans l'ensemble des travaux qu'ils produisent, on devrait

5 Karl Marx, Le Capital. Critique de l'économie politique [1867], dans Euvres, Économie I, Paris, Gallimard, coll. « La pléiade », 1965.

6 Claude Lévi-Strauss, Les structures élémentaires de la parenté, op. cit.

7 Edgar Morin, La méthode - 2. La Vie de la Vie, Paris, Éditions du Seuil, 1980, p. 94.

8 Philippe Moreau Defarges, La mondialisation, Paris, PUF, coll. «Que sais-je? », [1997] 2005, p. 28. 
régulièrement observer des questionnements éthiques, voir prescriptifs.

Si notre réflexion est juste, nous devrions, en examinant les travaux que les sciences humaines produisent, sur un objet quelconque, observer :

1) une intrication des propos;

2) un nombre réduit de catégories fondamentales;

3) une certaine conjugaison des discours analytique et éthique.

Si notre réflexion est erronée, nous devrions alors découvrir :

1) des propos exclusifs les uns des autres;

2) un nombre élevé de catégories analytiques;

3) une nette distinction des discours analytique et éthique.

\section{Méthodologie}

On pourrait vérifier ces hypothèses sur d'innombrables thématiques. Nous avons choisi de faire porter l'analyse sur les travaux de chercheurs qui se sont penchés sur des organismes internationaux, notamment sur l'Accord de libre-échange nord-américain (ALÉNA) ${ }^{9}$, le Commonwealth, la Coopération économique pour l'Asie-Pacifique (APEC) ${ }^{10}$, l'Organisation des États américains $(\mathrm{OÉA})^{11}$, l'Union africaine (UA) ${ }^{12}$, l'Union européenne (UE) ${ }^{13}$ et la Zone de libre-échange des Amériques (ZLÉA) ${ }^{14}$. Pour repérer ces travaux, nous avons recouru à trois index majeurs : ECONLIT, Worldwide Political Science Abstracts et Sociological Abstracts. Avec chacun d'eux, nous avons effectué une recherche sur le sigle anglais de l'organisme. Dans le cas de l'Inion africaine, nous avons aussi fait la recherche avec le sigle OAU, pour Organization of African Unity, parce que le changement de

\footnotetext{
$9 \quad$ North American Free Trade Agreement (NAFTA) en anglais.

10 Asia-Pacific Economic Cooperation (APEC) en anglais.

11 Organization of American States (OAS) en anglais.

12 African Union (AU) en anglais. Anciennement l'Organisation de l'Unité africaine (OUA) et Organization of African Unity (OAU) en anglais.

13 European Union (EU) en anglais.

14 Free Trade Area of Americas (FTAA) en anglais.
} 
dénomination est relativement récent; nous avons ensuite assimilé les deux entités. Pourquoi avoir choisi les organismes internationaux? Il n'y a pas de raison. Le choix du sujet nous semble arbitraire. De toute façon, cette recherche appellera forcément d'autres vérifications, sur d'autres sujets.

Pour veiller à ce que notre analyse ne prenne en considération que les publications qui ont quelque crédibilité dans le monde de la recherche, nous n'avons sélectionné que celles qui proviennent de revues au sein desquelles opère un comité de lecture. Nous avons par ailleurs préféré délimiter la période d'observation afin de rendre moins questionnable le rassemblement des textes; nous avons aussi voulu que notre regard s'attarde à des publications récentes. La période va donc de 1994 à 2007. Les trois portails de référencement que nous avons utilisés offrent des résumés de la plupart des articles. Ces résumés sont précieux parce qu'ils constituent des condensés des articles. Tous ces résumés, en outre, sont produits en anglais, quelle que soit la langue dans laquelle l'article a été écrit. Il devient alors possible de les traiter simultanément, sans distinction de langue, avec l'aide de logiciels d'analyse de données textuelles. À cet égard, nous avons employé Alceste dans sa version 4.9.

Dans le cas des articles qui ont été identifiés par les descripteurs (anglais) AU, APEC, Commonwealth, FTAA, NAFTA, OAS et OAU, nous avons retenu tous les titres qui comportaient un résumé. Si les articles avaient été référencés sous le descripteur (anglais) EU, nous avons intégré à l'ensemble tous ceux que relevait le Sociological Abstracts. ECONLIT et Worldwide Political Science Abstracts signalaient environ 5000 articles chacun sous le descripteur EU; nous nous sommes pourvus d'un échantillon aléatoire de 1000 notices dans chacun de ces deux portails, puis nous avons conservé ceux qui, parmi eux, affichaient un résumé. $\mathrm{Au}$ total, l'analyse repose sur un corpus de plus de 5000 résumés.

Pour des raisons de logistiques autant que pour des motifs analytiques, nous avons choisi d'examiner les organismes internationaux séparément les uns les autres. En procédant ainsi, nous 
avons été obligés d'exclure l'Union africaine parce que le logiciel de données textuelles ne parvenait pas à générer un minimum de deux classes qui pouvaient rester stables quand le corpus était entièrement examiné.

Pourquoi avoir choisi ces trois index que sont le Sociological Abstracts, ECONLIT et Worldwide Political Science Abstracts? Outre les qualités qui leur sont inhérentes et que nous avons déjà évoquées, outre le fait qu'ils jouissent d'une grande autorité, il nous a semblé que ce sont ceux au sein desquels il était le plus probable de repérer un grand nombre de publications qui portaient sur les organismes internationaux. Au plan méthodologique, de surcroît, nous avons pensé qu'il était indiqué de recourir à des portails qui appartiennent à des disciplines spécifiques et de vérifier si le portail lui-même était déterminant des contenus.

\section{Lexique de l'étude des organismes internationaux}

\subsection{Accord de libre-échange nord-américain}

En procédant à une analyse du corpus relatif à l'ALÉNA, le logiciel parvient à prendre en considération $79 \%$ de l'information qui est contenue dans l'ensemble des notices. Il répartit cette information dans quatre classes qui sont à peu près aussi importantes les unes que les autres. La première de ces classes contient $30 \%$ de l'information analysée. Le vocabulaire dominant est fortement économique : produit, exportation, taux, augmentation, prix, industrie, revenu, coût, croissance, échange, manufacture, marché $^{15}$. La deuxième classe comprend $26 \%$ des données qui sont prises en considération. Les propos qui y sont tenus sont à une distance relativement nette de ceux qui valent pour la première classification. Ils se déploient autour des idées de société et de justice. Ils sont marqués de mots comme : mouvement,

15 Product $+\left(\chi^{2}=139\right)$, export+ $\left(\chi^{2}=126\right)$, rate $+\left(\chi^{2}=124\right)$, increase + $\left(\chi^{2}=110\right)$, price $+\left(\chi^{2}=93\right)$, industr $+\left(\chi^{2}=82\right)$, wage $+\left(\chi^{2}=79\right)$, income+ $\left(\chi^{2}=77\right)$, cost $\left(\chi^{2}=74\right)$, growth $\left(\chi^{2}=68\right)$, exchange+ $\left(\chi^{2}=66\right)$, manufactur + $\left(\chi^{2}=66\right)$, market+ $\left(\chi^{2}=46\right)$, tariff $+\left(\chi^{2}=35\right)$. 
social, global, transnational, néolibéral, organisme, travail, réseau, société, civil ${ }^{16}$; un peu plus loin dans la hiérarchie du vocabulaire, on trouve aussi des termes comme activiste, classe, genre, femme, droit, féminisme, justice ${ }^{17}$. La troisième classe porte sur $22 \% \mathrm{du}$ discours appréhendé. Elle se distingue nettement de la première. Elle est plus spécifique par rapport à la deuxième en ce qu'elle est plus politique que sociale. Son lexique est émaillé de mots comme : international, environnemental, gouvernement, législation, institutionnel, légal, fédéral, clause, intergouvernemental, régime, constitutionnel ${ }^{18}$. La quatrième classe est en proportion égale à la précédente. Elle ne se distingue de la première qu'en tant qu'elle y ajoute une dimension géographique. Les mots libre, échange et entente ${ }^{19}$ témoignent de sa composante économique, tandis que les termes nord, régional, Amérique, Asie, multilatéral, Japon, est, pays, Caraïbe, latin, sud-est ${ }^{20}$ soulignent sa dimension géopolitique.

$\overline{16}$ Mouvement+ $\left(\chi^{2}=236\right)$, social+ $\left(\chi^{2}=201\right)$, global+ $\left(\chi^{2}=173\right)$, transnational+ $\left(\chi^{2}=159\right)$, neolibéral+ $\left(\chi^{2}=114\right)$, organ $+\left(\chi^{2}=110\right)$, labor+ $\left(\chi^{2}=89\right)$, network $+\left(\chi^{2}=78\right)$, societ $\left(\chi^{2}=76\right)$, civil $\left(\chi^{2}=76\right)$.

17 Class $+\left(\chi^{2}=59\right)$, gender $+\left(\chi^{2}=54\right)$, women $\left(\chi^{2}=48\right)$, right $\left(\chi^{2}=48\right)$, feminist $+\left(\chi^{2}=45\right)$, justice $\left(\chi^{2}=45\right)$.

18 International $+\left(\chi^{2}=98\right)$, environmental $+\left(\chi^{2}=93\right)$, govern $+\left(\chi^{2}=76\right)$, legisl $\left(\chi^{2}=65\right)$, institutional $\left(\chi^{2}=64\right)$, legal $+\left(\chi^{2}=55\right)$, fedéral $+\left(\chi^{2}=52\right)$, clause+ $\left(\chi^{2}=51\right)$, intergovernmental $\left(\chi^{2}=51\right)$, regime+ $\left(\chi^{2}=47\right)$, constitutional $\left(\chi^{2}=47\right)$.

19 Free+ $\left(\chi^{2}=359\right)$, trade $+\left(\chi^{2}=333\right)$ et agreement+ $\left(\chi^{2}=289\right)$.

20 North $\left(\chi^{2}=176\right)$, regional+ $\left(\chi^{2}=145\right)$, america $+\left(\chi^{2}=84\right)$, asia $\left(\chi^{2}=122\right)$, multilateral $+\left(\chi^{2}=105\right)$, japan $+\left(\chi^{2}=103\right)$, east $\left(\chi^{2}=59\right)$, countries $\left(\chi^{2}=58\right)$, caribbean $\left(\chi^{2}=57\right)$, latin $\left(\chi^{2}=53\right)$, south-east $\left(\chi^{2}=35\right)$. 


\section{Tableau 1}

\section{Exemples d'extraits où se manifestent des éléments du lexique} pour chacune des classes qui se dégagent du corpus sur l'ALÉNA

Classe 1 - Économie "[...] findings indicate that downstream-consuming firms that diversify production in NAFTA countries avert some trade policy risk associated with higher steel costs caused by safeguard protection $^{21}$."

Classe 2 - Social

"The paper details innovative strategies of local labor organizers to unionize workers under the hostile conditions surrounding export processing zones. The case study from the Philippines outlines a comprehensive, scalar strategy with an analysis of four key elements: first, the local political context ...22"

Classe 3 - Politique

"There are indications that the elevated level of international environmental interest might have passed the midpoint, in the nineteen nineties, of a five-decade period ${ }^{23}$. "

Classe 4 - Géographie

"Modeling the spatial economy of Brazil using the usual assumptions of CGE models makes little sense when one state, Sao Paulo, accounts for $40 \%$ of GDP and where transportation costs are high and accessibility low compared to European or North American standards ${ }^{24}$."

\subsection{Union européenne}

Quand elle s'intéresse aux notices relatives à l'Union européenne, l'analyse parvient à saisir $78 \%$ des unités élémentaires qui s'offrent à elle. Cette fois, elle repère sept classes. Aucune d'entre elles n'est nettement dominante : elles s'approprient chacune

21 Benjamin H. Liebman et Kasaundra M. Tomlin, "Steel Safeguards and the Welfare of U.S. Steel Firms and Downstream Consumers of Steel: A Shareholder Wealth Perspective ", Canadian Journal of Economics, vol. 40, ${ }^{\circ}$ 3, août 2007, p. 812-842.

22 Steven C. McKay, "The Squeaky Wheel's Dilemma: New Forms of Labor Organizing in the Philippines ", Labor Studies Journal, vol. 30, $\mathrm{n}^{\circ} 4$, hiver 2006, p. 41-63.

23 David J. LePoire, "Logistic Analysis of Recent Environmental Interest ", Technological Forecasting and Social Change, vol. 73, n 2, février 2006, p. 153167.

24 Eduasdo A. Haddad et Geoffrey J. D. Hewings, "Market Imperfections in a Spatial Economy: Some Experimental Results ", Quarterly Review of

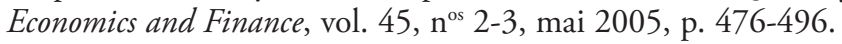


entre 20 et $11 \%$ du corpus retenu. La sémantique de l'ensemble de ces classes est semblable à celle qui vaut pour l'ALÉNA, sauf qu'elle est plus détaillée. On y trouve, à nouveau, une oscillation entre la sociopolitique, d'une part, et l'économique, d'autre part. La toute première catégorie à être générée se développe sur un lexique qui contient des termes comme produit, échange, agriculture, industrie, cồt, marché, exportation, investissement, secteur, prix, tarif, compétition ${ }^{25}$. La deuxième a trait à la connaissance et à la science; son vocabulaire caractéristique comprend des unités comme information, recherche, réseau, science, méthode, technologie, connaissance ${ }^{26}$. La troisième est de l'ordre du politicoculturel; on y lit des termes comme discours, culturel, identité, politique, culture $^{27}$. La quatrième conduit vers des considérations sociales; on y trouve des marqueurs comme : femme, famille, emploi, chômage, travail ${ }^{8}$. La cinquième soulève les questions de sécurité; sécurité, étranger, guerre, OTAN, défense, militaire ${ }^{29}$, en sont des constituants caractéristiques. La sixième se tient à courte distance de la première, qui est d'ordre économique, mais elle en spécifie une dimension comptable; elle est composée de mots comme monétaire, fiscal, inflation, euro, taux, budget, banque ${ }^{30}$. La septième ajoute à l'ensemble son aspect politique; des termes comme élection, parlement, élite, démocratie, électoral, référendum ${ }^{31}$ y sont fréquents.

25 Product $+\left(\chi^{2}=455\right)$, trade $+\left(\chi^{2}=351\right)$, agricultural $\left(\chi^{2}=343\right)$, industr + $\left(\chi^{2}=241\right)$, cost $\left(\chi^{2}=223\right)$, market $\left(\chi^{2}=189\right)$, export $+\left(\chi^{2}=189\right)$, invest + $\left(\chi^{2}=180\right)$, sector $+\left(\chi^{2}=177\right)$, price $+\left(\chi^{2}=168\right)$, tariff $+\left(\chi^{2}=155\right)$, competition $+\left(\chi^{2}=147\right)$.

26 Inform $+\left(\chi^{2}=158\right)$, research $+\left(\chi^{2}=147\right)$, network $+\left(\chi^{2}=117\right)$, scientific $\left(\chi^{2}=110\right)$, méthode $\left(\chi^{2}=92\right)$, technolog+ $\left(\chi^{2}=91\right)$, knowledge $\left(\chi^{2}=86\right)$.

27 Discourse $+\left(\chi^{2}=348\right)$, cultural+ $\left(\chi^{2}=307\right)$, identity $\left(\chi^{2}=256\right)$, polit + $\left(\chi^{2}=236\right)$, culture+ $\left(\chi^{2}=190\right)$.

28 Woman $+\left(\chi^{2}=339\right)$, famil+ $\left(\chi^{2}=327\right)$, employ+ $\left(\chi^{2}=316\right)$, unemploy+ $\left(\chi^{2}=301\right)$, work $\left(\chi^{2}=256\right)$.

29 Securit+ $\left(\chi^{2}=546\right)$, foreign $+\left(\chi^{2}=280\right)$, war+ $\left(\chi^{2}=258\right)$, NATO $\left(\chi^{2}=256\right)$, defense+ $\left(\chi^{2}=255\right)$, militar+ $\left(\chi^{2}=219\right)$.

30 Monetar+ $\left(\chi^{2}=698\right)$, fiscal $+\left(\chi^{2}=513\right)$, inflation $+\left(\chi^{2}=436\right)$, euro+ $\left(\chi^{2}=297\right)$, rate $+\left(\chi^{2}=284\right)$, budget $+\left(\chi^{2}=232\right)$, bank $\left(\chi^{2}=205\right)$.

31 Election $+\left(\chi^{2}=253\right)$, parliament $+\left(\chi^{2}=194\right)$, elite $\left(\chi^{2}=153\right)$, democrat+ $\left(\chi^{2}=121\right)$, electoral $\left(\chi^{2}=106\right)$, referemdum $\left(\chi^{2}=90\right)$. 


\section{Tableau 2}

\section{Exemples d'extraits où se manifestent des éléments du lexique pour chacune des classes qui se dégagent du corpus sur l'UE}

Classe 1 - Économie

"In transition economies knowledge transfer is subject to several sources of market failure which provides a justification for government intervention. The success of these policies also depends upon the ability of academic institutions \& the business sector to collaborate in technology networks, innovation clusters \& the mobility of researchers between the two sectors $^{32}$."

Classe 2 - Connaissance - science

"This article provides a theoretically informed and empirically grounded explanation of the policy reform by evaluating the analytical leverage of three integration theories, mainly by relying on two methods - process tracing and congruence procedure - employing a variety of primary and secondary sources $^{33}$. "

Classe 3 - Politicoculturel

"We explore our informants' discourses as mediated by the historical context of common experience (eviction, displacement, communism) pertaining mostly to the older generation $\&$ by the cultural-economic context (shopping, entertainment) largely in the case of our younger informants ${ }^{34}$."

Classe 4 - Social

"The Belgian case emphasizes the negative role of the EU in retrenching pension rights and benefits for women. The Swedish case is interesting because of the scope of its reform and the fact that women in the service sector end up supporting the principle of tying benefits to contributions ${ }^{35}$."

Classe 5 - Sécurité

"Moreover, the current preoccupation with the war on terror has encouraged the co-option of development resources for security functions resulting in the incremental securitisation of development policies ${ }^{36}$. »

32 Will Bartlett et Nevenka Cuckovic, "Knowledge Transfer, Institutions, and Innovation in Croatia and Slovenia ", Drustvena Istrazivanja, vol. 15, $\mathrm{n}^{\circ} 3$, mai-juin 2006, p. 371-399.

33 Lucia Quaglia, «The politics of financial services regulation and supervision reform in the European Union ", European Journal of Political Research, vol. $46, \mathrm{n}^{\circ} 2$, mars 2007 , p. 269-290.

34 Aleksandra Galasinska et Dariusz Galasinski, «Shopping for a New Identity: Constructions of the Polish-German Border in a Polish Border Community ", Ethnicities, vol. 5, no 4, décembre 2005, p. 510-529.

35 Patrik Marier, "Affirming, Transforming, or Neglecting Gender? Conceptualizing Gender in the Pension Reform Process ", Social Politics, vol. 14, nº 2, été 2007, p. 182-211.

36 Susan Willett, "New Barbarians at the Gate: Losing the Liberal Peace in 
Classe 6 - Comptabilité

"With this has come tight monetary rules via a constitution that favors low inflationary rates, much of which was via the machinations of the EMU, which is obsessed with price regulation $^{37}$. "

Classe 7 - Politique

"The Referendum coincided with the 2004 local government elections and campaigns by immigrant organizations to promote political responsiveness to immigrant non-citizens (including asylum seekers) entitled to vote in local government elections ${ }^{38}$."

\subsection{Zone de libre-échange des Amériques}

L'ensemble des notices sur la ZLÉA ne permet à l'analyse d'identifier que trois classes qui, ensemble, réunissent $79 \%$ de l'information disponible. La première des catégories est plus imposante que les autres : elle n'absorbe pas moins de $63 \%$ de l'information appréhendée. Elle est d'une sémantique composite où se conjuguent des terminologies relatives aux nations (Etats-Unis, Canada, Etats, Caraïbes $^{39}$ ), à la politique (négociation, politique, gouverne$\left.m e n t^{40}\right)$, à la philosophie (développement, progres ${ }^{41}$ ) et à la philosophie politique (entente, coopération, pouvoir, arrangement ${ }^{42}$ ). La deuxième catégorie capte $27 \%$ du corpus accessible. Elle est très analytique (résultats, modèle, estimation, effet, équilibre ${ }^{43}$ ), mais elle a essentiellement pour objet l'univers de l'économie (gain, augmentation, exportation, production, tarif, secteur $\left.{ }^{44}\right)$, auquel

Africa ", Review of African Political Economy, vol. 32, n 106, décembre 2005, p. $569-594$.

37 Christoph Hermann, "Neoliberalism in the European Union ", Studies in Political Economy, n $^{\circ}$ 79, printemps 2007, p. 61-89

38 Bryan Fanning et Fidele Mutwarasibo, " Nationals/non-nationals: Immigration, citizenship and politics in the Republic of Ireland ", Ethnic and Racial Studies, vol. 30, $\mathrm{n}^{\circ} 3$, mai 2007, p. 439-460.

39 US $\left(\chi^{2}=11\right)$, Canada $+\left(\chi^{2}=10\right)$, state+ $\left(\chi^{2}=7\right)$, Caribbean $\left(\chi^{2}=6\right)$.

40 Negoti+ $\left(\chi^{2}=21\right)$, polit $+\left(\chi^{2}=18\right)$, government $+\left(\chi^{2}=6\right)$.

41 Develop $+\left(\chi^{2}=10\right)$, progress $\left(\chi^{2}=7\right)$.

42 Agreement $+\left(\chi^{2}=8\right)$, cooperat $+\left(\chi^{2}=8\right)$, power $\left(\chi^{2}=8\right)$, arrangement+ $\left(\chi^{2}=8\right)$.

43 Result $+\left(\chi^{2}=59\right)$, model $+\left(\chi^{2}=55\right)$, estimate $+\left(\chi^{2}=54\right)$, effect $+\left(\chi^{2}=38\right)$, equilibrium $\left(\chi^{2}=38\right)$.

44 Gain $+\left(\chi^{2}=44\right)$, increase $+\left(\chi^{2}=40\right)$, export $+\left(\chi^{2}=38\right)$, product $+\left(\chi^{2}=35\right)$, tariff $\left(\chi^{2}=31\right)$, sector $+\left(\chi^{2}=31\right)$. 
s'associe parfois celui du bien-être (bien-être, emploi, service ${ }^{45}$ ). La troisième catégorie concerne $10 \%$ du corpus analysable. Elle met en évidence les préoccupations d'ordre social (transnational, mouvement, civil, démocratie, action, activiste, réseau, collective, classe, résistance, genre, proteste $\left.{ }^{46}\right)$.

\section{Tableau 3}

\section{Exemples d'extraits où se manifestent des éléments du lexique pour chacune des classes qui se dégagent du corpus sur la ZLÉA}

Classe 1 - Nation - politique - philosophie " "Spoke-spoke" systems include the Assoc. of Caribbean States (ACS) \& agreements with Mexico, Colombia, \& Venezuela. Although CARICOM has made significant economic progress, the region needs satellite offices to decentralize the integration process, combined with better coordination, expansion of financial support, \& improved information systems architecture $^{47}$."

Classe 2 - Analytique - économie - bien-être

"The FTAA formation is simulated, and its impacts upon poverty and income distribution in Brazil analyzed. Results point to the fact that even a large shock like the one simulated wouldn't have a strong impact on poverty reduction in Brazil, although the results are concentrated on the poorest households ${ }^{48}$."

Classe 3 - Social

" [...] the influence of civil society actors in regionalist governance in the Americas is extremely limited. This is due to persistent institutional barriers to inclusion, the practical obstacles for many groups of scaling up to the regional/transnational level and the particular difficulties associated with accessing tradebased negotiations ${ }^{49}$."

45 Welfare $\left(\chi^{2}=45\right)$, employ+ $\left(\chi^{2}=25\right)$, service+ $\left(\chi^{2}=23\right)$.

46 Transnational $+\left(\chi^{2}=127\right)$, movement $+\left(\chi^{2}=126\right)$, civil $\left(\chi^{2}=82\right)$, démocract $\left(\chi^{2}=71\right)$, action $+\left(\chi^{2}=63\right)$, activism $\left(\chi^{2}=44\right)$, network $\left(\chi^{2}=42\right)$, collective+ $\left(\chi^{2}=35\right)$, class $\left(\chi^{2}=35\right)$, resistance $\left(\chi^{2}=32\right)$, gender $+\left(\chi^{2}=27\right)$, protest + $\left(\chi^{2}=27\right)$.

47 Shelton Nicholls, Anthomny Birchwood, Philip Colthrust et Earl Boodoo, "The State of and Prospects for the Deepening and Widening of Caribbean Intregration ", World Economy, vol. 23, n 9, septembre 2000, p. 11611194.

48 Ferreira Filho, Bento de Souza, Joaquim Mark et Jonathan Horridge, "Economic Integration, Poverty and Regional Inequality in Brazil ", Revista Brasileira de Economia, vol. 60, n 4, octobre-décembre 2006, p. 363-387.

49 Jean Grugel, "Regionalist Governance and Transnational Collective Action in Latin America ", Economy and Society, vol. 35, n 2, mai 2006, p. 209231. 


\subsection{Organisation des États américains}

En se penchant sur les notices relatives à l'Organisation des États américains, l'analyse retient $66 \%$ de tout ce qui ce qu'elles renferment. Cela signifie d'entrée de jeu, qu'une proportion relativement importante du corpus est éclatée, difficilement pénétrable. Quatre catégories sont construites. Sur $100 \%$ du total explorable, $16 \%$ des unités élémentaires constituent la première classe. Cette classe est la plus petite des quatre. Elle est de l'ordre du politico-économique regroupant des mots comme échange, gouvernement, entente, négociation, société, libre, civil, représentation ${ }^{50}$. Trente-huit pourcent (38\%) des unités élémentaires constituent la deuxième classe, laquelle s'intéresse aux aspects politicosociaux : au premier chef, à la démocratie (démo$c r a c+e t$ démocrat ${ }^{51}$ ); ensuite, à la géopolitique (américain, États-Unis, États, région, Pérou ${ }^{52}$ ); puis, à la sécurité (défense ${ }^{53}$ ); enfin au social (organisation, solidarité, élection ${ }^{54}$ ). Vingt pour cent $(20 \%)$ des unités composent la troisième classe, laquelle se présente dans un triple vocabulaire où se joignent des termes relatifs à l'analytique (étude, valide, rapport, enquête, résultats, échantillon, données ${ }^{55}$ ), à la déviance (drogue, abus, alcool, contrevenant, substance, ecstasy ${ }^{56}$ ) et à l'ethnie (latino, noir, religion, blanc, racial $\left.{ }^{57}\right)$. Vingt-six pourcent $(26 \%)$ des unités forment la quatrième classe. Cette classe propose un vocabulaire qui est le signe de préoccupations sociales (droits, humain, trafic, indigène,

$50 \quad$ Trade $\left(\chi^{2}=78\right)$, govern $+\left(\chi^{2}=56\right)$, agreement $+\left(\chi^{2}=49\right)$, negotiation + $\left(\chi^{2}=44\right)$, society+ $\left(\chi^{2}=43\right)$, free $\left(\chi^{2}=43\right)$, civil+ $\left(\chi^{2}=35\right)$, represent+ $\left(\chi^{2}=32\right)$.

51 Démocrac+ $\left(\chi^{2}=61\right)$ et démocrat+ $\left(\chi^{2}=48\right)$.

52 American $+\left(\chi^{2}=42\right)$, US $\left(\chi^{2}=18\right)$, states $+\left(\chi^{2}=17\right)$, region $+\left(\chi^{2}=15\right)$, Peru $\left(\chi^{2}=9\right)$.

53 Defense $\left(\chi^{2}=15\right)$.

54 Organisation $\left(\chi^{2}=13\right)$, solidarité $\left(\chi^{2}=12\right)$, élection $\left(\chi^{2}=10\right)$.

55 Stud $+\left(\chi^{2}=35\right)$, valid $+\left(\chi^{2}=28\right)$, report $+\left(\chi^{2}=27\right)$, survey+ $\left(\chi^{2}=24\right)$, findings $\left(\chi^{2}=23\right)$, sample $\left(\chi^{2}=19\right)$, data $\left(\chi^{2}=16\right)$.

56 Drug+ $\left(\chi^{2}=80\right)$, abuse $+\left(\chi^{2}=42\right)$, alcohol $\left(\chi^{2}=36\right)$, offender $+\left(\chi^{2}=24\right)$, substance $\left(\chi^{2}=24\right)$, ecstasy $\left(\chi^{2}=16\right)$.

57 Latino+ $\left(\chi^{2}=28\right)$, black $+\left(\chi^{2}=24\right)$, reli+ $\left(\chi^{2}=20\right)$, white $\left(\chi^{2}=20\right)$, racial+ $\left(\chi^{2}=15\right)$. 
femmes, enfant, pays, travail, migration, culture $\left.^{58}\right)$.

\section{Tableau 4}

Exemples d'extraits où se manifestent des éléments du lexique pour chacune des classes qui se dégagent du corpus sur l'OÉA

Classe 1 - Politico-économique

"The new free trade agreements are rescaling governance in ways that have critical implications for subnational governments. The nation-state is not simply being hollowed out; rather, a new governance nexus is forming - of nation-states, multinational corporations, \& international agreements - which explicitly excludes subnational \& local government voice ${ }^{59}$."

Classe 2 - Politicosocial

"Over time, the Organization of American States has become institutionally and normatively more capable of defending democracy in the region. Yet the OAS is as selective in its interventions on behalf of democratic promotion today as it was in the early nineteen nineties ${ }^{60}$."

Classe 3 - Analytique - déviance - ethnie

"Many analysts have argued that the most harmful forms of drug use are disproportionately concentrated in poor communities of color, \& that this pattern - combined with law enforcement's tendency to focus on outdoor drug activity - explains racial disparity in drug arrests. Others contend that comparatively high Black \& Latino drug arrest rates result from racial bias or racist intent on the part of the architects or lieutenants of the drug war $^{61}$."

Classe 4 - Social

«Local political struggles $\&$ acts of resistance by disenfranchised groups in Mexico offer insight to understand the impact of indigenous \& other social movements in furthering human rights $^{62}$.»

58 Right $+\left(\chi^{2}=75\right)$, human $\left(\chi^{2}=68\right)$, traffick $+\left(\chi^{2}=38\right)$, indigenous $\left(\chi^{2}=29\right)$, women $+\left(\chi^{2}=29\right)$, child $\left(\chi^{2}=28\right)$, countr+ $\left(\chi^{2}=24\right)$, labour $\left(\chi^{2}=23\right)$, migration $+\left(\chi^{2}=23\right)$, cultural $\left(\chi^{2}=20\right)$.

59 Mildred Warner et Jennifer Gerbasi, "Rescaling and Reforming the State under NAFTA: Implications for Subnational Authority ", dans International Journal of Urban and Regional Research, vol. 28, n 4, décembre 2004, p. 858873.

60 Craig Arceneaux et David Pion-Berlin, «Issues, Threats, and Institutions: Explaining OAS Responses to Democratic Dilemmas in Latin America ", Latin American Politics and Society, vol. 49, n 2, été 2007, p. 1-31.

61 Katherine Beckett, Kris Nyrop, Lori Pfingst et Melissa Bowen, «Drug Use, Drug Possession Arrests, and the Question of Race: Lessons from Seattle ", Social Problems, vol. 52, n 3, août 2005, p. 419-441.

62 Linda Lopez, «Advancing Human Rights Policy: Does Grassroots Mobilization and Community Dispute Resolution Matter? Insights from Chiapas, Mexico ", The Review of Policy Research, vol. 22, $\mathrm{n}^{\circ}$ 1, janvier 2005, 


\subsection{Coopération économique pour l'Asie-Pacifique}

Quand l'analyse se tourne vers les notices relatives à l'APEC, elle s'empare de $78 \%$ du corpus et construit une typologie à quatre composants. Le premier, comportant $24 \%$ des unités élémentaires abordables, est nettement d'ordre social : les mots migrant, transnational, contexte, travail, droit, manifestation, humain, mouvement, femmes, genre ${ }^{63}$ sont typiques de son lexique. Le deuxième, lié à $31 \%$ du corpus analysable, se veut géopolitique à un niveau plutôt général. Il se définit à travers des mots comme Asie, pacifique, est, régional, Europe, asiatique, région ${ }^{64}$, d'une part, et des mots comme coopération, establishment, forum, intégration, projet, institut, souveraineté ${ }^{65}$, d'autre part. Le troisième, comprenant $13 \%$ des unités élémentaires retenues, est aussi d'ordre géopolitique. Il est relativement abstrait dans sa dimension politique, évoquant des notions comme république, relation, mutualité ${ }^{66}$, mais il est plus spécifique en ce qu'il identifie des nations (Chine, Australie, Etats-Unis, Inde, Japon ${ }^{67}$ ). Le dernier, attaché à $32 \%$ du corpus envisageable, se rapporte à l'économie. Il manipule des termes comme échange, produit, croissance, libérale, exportation, secteur, augmentation, service, gain, fluidité, industrie, investissement ${ }^{68}$.

p. 77-92.

63 Migrant $\left(\chi^{2}=60\right)$, transnational $\left(\chi^{2}=53\right)$, context $+\left(\chi^{2}=51\right)$, labor + $\left(\chi^{2}=47\right)$, right $+\left(\chi^{2}=45\right)$, protest $+\left(\chi^{2}=43\right)$, human $\left(\chi^{2}=37\right)$, movement+ $\left(\chi^{2}=36\right)$, women $+\left(\chi^{2}=36\right)$, gender $+\left(\chi^{2}=35\right)$.

64 Asia $+\left(\chi^{2}=229\right)$, pacific $+\left(\chi^{2}=141\right)$, east $\left(\chi^{2}=96\right)$, regional $+\left(\chi^{2}=95\right)$, Europe+ $\left(\chi^{2}=61\right)$, asian $+\left(\chi^{2}=48\right)$, région $\left(\chi^{2}=37\right)$.

65 Cooperat+ $\left(\chi^{2}=81\right)$, establish $+\left(\chi^{2}=35\right)$, forum $+\left(\chi^{2}=30\right)$, integrat + $\left(\chi^{2}=27\right)$, project $+\left(\chi^{2}=25\right)$, institut $+\left(\chi^{2}=24\right)$, souvereign $\left(\chi^{2}=23\right)$.

66 Republic $\left(\chi^{2}=59\right)$, relation $+\left(\chi^{2}=55\right)$, mutual $+\left(\chi^{2}=40\right)$.

67 China $+\left(\chi^{2}=96\right)$, Austral+ $\left(\chi^{2}=67\right)$, US $\left(\chi^{2}=38\right)$, India $\left(\chi^{2}=33\right)$, Japan + $\left(\chi^{2}=24\right)$.

68 Trade $\left(\chi^{2}=86\right)$, product $+\left(\chi^{2}=61\right)$, growth $\left(\chi^{2}=46\right)$, liberal+ $\left(\chi^{2}=44\right)$, export+ $\left(\chi^{2}=44\right)$, sector $\left(\chi^{2}=43\right)$, increase+ $\left(\chi^{2}=42\right)$, service+ $\left(\chi^{2}=39\right)$, gain $+\left(\chi^{2}=38\right)$, flow $+\left(\chi^{2}=35\right)$, industr $+\left(\chi^{2}=35\right)$, invest $+\left(\chi^{2}=34\right)$. 


\section{Tableau 5}

\section{Exemples d'extraits où se manifestent des éléments du lexique pour chacune des classes qui se dégagent du corpus sur l'APEC}

Classe 1 - Social

" $[\ldots]$ an analysis of the labor-receiving state's practices toward migrant women domestic workers in Malaysia. I ascertain that the exercise of a particular kind of state power as evinced from policies \& legislation, consistently make visible migrant women's presence in society even as their labor in households is rendered invisible ${ }^{69}$."

Classe 2 - Géopolitique générale

" [...] behind East Asian regionalism is nationalism, which constitutes a driving force for regionalism; that two competing orders (Asia-Pacific regionalism vs pan-Asianism) create different expectations \& visions of how East Asia region should evolve \& they are in tension \& lead to different directions; \& that East Asia lacks a convincing \& acceptable normative framework $^{70}$."

Classe 3 - Géopolitique spécifique

" Early in 2000, Australian bilateral relations with US, Japan, the People's Republic of China, \& Indonesia \& regional \& multinational forums were revisited, but with an old foreign policy logic \& ambivalences on the part of the Howard government $^{71}$.»

Classe 4 - Économique

"The relationship between these indicators and trade flows is estimated using a gravity model that includes tariffs and other standard variables. Enhanced port efficiency has a large and positive effect on trade flows. Regulatory barriers deter trade. Improvements in customs and greater e-business use significantly expand trade but to a lesser degree than improvements in ports or regulations ${ }^{72}$."

\subsection{Commonwealth}

Après avoir examiné le corpus sur le Commonwealth, l'analyse construit sept catégories qui comprennent $79 \%$ de l'information

69 Christine B. N. Chin, «Visible Bodies, Invisible Work: State Practices toward Migrant Women Domestic Workers in Malaysia ", Asian and Pacific Migration Journal, vol. 12, $\mathrm{n}^{\text {os }} 1-2,2003$, p. 49-73.

70 Baogang He, "East Asian Ideas of Regionalism: A Normative Critique ", Australian Journal of International Affairs, vol. 58, $\mathrm{n}^{\circ} 1$, mai 2004, p. 105125.

71 Michael Wesley, « Perspectives on Australian Foreign Policy, 2001 », Australian Journal of International Affairs, vol. 56, n 1, avril 2002, p. 47-63.

72 John S. Wilson, Catherine L. Mann et Tsunehiro Otsuki, «Trade Facilitation and Economic Development: A New Approach to Quantifying the Impact ", World Bank Economic Review, vol. 17, n 3, 2003, p. 367-389. 
disponible. Le poids de ces catégories varie entre 22 et $7 \%$. La première est nettement analytique (recherche, donnée, enquête, échantillon, découverte, étude, facteur, varier, rapport ${ }^{73}$ ) et elle a pour objet des questions sociales (femmes, santé, éducation, enseignants, hommes, violence, abus, étudiants, enfant, risque $\left.{ }^{74}\right)$. La deuxième est économique (service, cô̂t, marché, privé, fond, assurance, prix, taxe, bénéfice $\left.{ }^{75}\right)$. La troisième a trait à la sécurité (sécurité, guerre, étranger, terrorisme, indépendance, nucléaire, militaire $^{76}$ ) et à la géographie (Asie, Britannique, Europe, Russie, Australie, pacifique $e^{77}$ ). La quatrième renvoie aux questionnements juridiques et, à un moindre degré, sociaux; on y lit des mots comme : lois, droit, légal, citoyen, souverain, peuple, racial, indigène, tolérance, exclusion, racisme, liberté ${ }^{78}$. La cinquième porte sur la politique; en constitue les jalons des mots comme élection, président, vote, parlement, campagne, voté, ministre, candidats ${ }^{79}$. La sixième catégorie est d'ordre historique; les références aux périodes (dix-neuvième, années mille neuf cent, centenaires, vingtième, dix-septième, début ${ }^{80}$ ) et aux événements (décolonisation, accord,

$\overline{73}$ Research $+\left(\chi^{2}=334\right)$, data $\left(\chi^{2}=324\right)$, interview+ $\left(\chi^{2}=214\right)$, sample+ $\left(\chi^{2}=176\right)$, findings $\left(\chi^{2}=163\right)$, study+ $\left(\chi^{2}=153\right)$, factors $\left(\chi^{2}=90\right)$, vari+ $\left(\chi^{2}=88\right)$, report+ $\left(\chi^{2}=88\right)$.

74 Women $+\left(\chi^{2}=295\right)$, health $+\left(\chi^{2}=284\right)$, educat $+\left(\chi^{2}=123\right)$, teachers + $\left(\chi^{2}=123\right)$, men $+\left(\chi^{2}=98\right)$, violence $+\left(\chi^{2}=79\right)$, abuse+ $\left(\chi^{2}=77\right)$, students + $\left(\chi^{2}=77\right)$, child $\left(\chi^{2}=69\right)$, risk $+\left(\chi^{2}=66\right)$.

75 Service $+\left(\chi^{2}=380\right)$, cost $\left(\chi^{2}=304\right)$, market $+\left(\chi^{2}=237\right)$, private+ $\left(\chi^{2}=207\right)$, fund $+\left(\chi^{2}=200\right)$, insur+ $\left(\chi^{2}=181\right)$, price+ $\left(\chi^{2}=179\right)$, tax $\left(\chi^{2}=167\right)$, benefit+ $\left(\chi^{2}=158\right)$.

76 Securit+ $\left(\chi^{2}=228\right)$, war $\left(\chi^{2}=222\right)$, foreign $+\left(\chi^{2}=201\right)$, terror $+\left(\chi^{2}=170\right)$, independent $\left(\chi^{2}=163\right)$, nuclear $\left(\chi^{2}=78\right)$, militar+ $\left(\chi^{2}=76\right)$.

77 Asia $+\left(\chi^{2}=183\right)$, British $+\left(\chi^{2}=149\right)$, Europe+ $\left(\chi^{2}=148\right)$, Russia $\left(\chi^{2}=147\right)$, Austral+ $\left(\chi^{2}=147\right)$, pacifict $\left(\chi^{2}=107\right)$.

78 Law $\left(\chi^{2}=554\right)$, right $+\left(X^{2}=521\right)$, legal $+\left(\chi^{2}=249\right)$, citizen $+\left(\chi^{2}=159\right)$, sovereign $+\left(\chi^{2}=149\right)$, people+ $\left(\chi^{2}=134\right)$, racial $+\left(\chi^{2}=114\right)$, indigenous + $\left(\chi^{2}=112\right)$, toler $+\left(\chi^{2}=106\right)$, exclusion $+\left(\chi^{2}=98\right)$, racism $+\left(\chi^{2}=83\right)$, freedom $+\left(\chi^{2}=69\right)$.

79 Elect $+\left(\chi^{2}=1096\right)$, president $+\left(\chi^{2}=330\right)$, vote $+\left(\chi^{2}=292\right)$, parliament + $\left(\chi^{2}=185\right)$, campaign $+\left(\chi^{2}=165\right)$, voter $+\left(\chi^{2}=149\right)$, minister $+\left(\chi^{2}=141\right)$, candidates+ $\left(\chi^{2}=98\right)$.

80 Nineteen $\left(\chi^{2}=637\right)$, nineties+ $\left(\chi^{2}=439\right)$, centur+ $\left(\chi^{2}=245\right)$, twentieth $\left(\chi^{2}=165\right)$, nineteenth $\left(\chi^{2}=160\right)$, begin $\left(\chi^{2}=49\right)$. 
fédération ${ }^{81}$ y sont courantes. La septième se rapporte aux théories politicosociales (politique, article, contemporanéité, théorie, concepts, féminin, société, pensée, discours, dimension, mainstream, critique, approche $\left.e^{82}\right)$.

\section{Tabelau 6}

\section{Exemples d'extraits où se manifestent des éléments du lexique pour chacune des classes qui se dégagent du corpus sur le Commonwealth}

Classe 1 - Analyticosocial

"We examine the effects of employment-contingent health insurance (ECHI) on married women's labor supply following a health shock. First, we develop a theoretical framework that examines the effects of ECHI on the labor supply response to a health shock, which suggests that women with ECHI are less likely to reduce their labor supply in response to a health shock, relative to women with health insurance through their spouse's employer $^{83}$."

Classe 2 - Économie

"We show that an individual mandate, and an employer mandate combined with an individual mandate, both would yield universal coverage with a relatively small increase in government costs relative to state gross domestic product and current health spending ${ }^{84}$."

Classe 3 - Sécurité - géographie

"Liberal governments trying to adapt to a rapidly changing international environment have been mindful of Australian interests, \& especially the ongoing search for security. Adapting to declining British power, to militant communism in East \& Southeast Asia, \& to a new, under-defined alliance with the United States was no easy task. The Colombo Plan for aid to South \& Southeast Asia \& the dismantling of the White Australia Policy represented two forms of positive engagement with Asia ${ }^{85}$."

81 Settlement+ $\left(\chi^{2}=61\right)$, decolonizati $\left(\chi^{2}=23\right)$, federation $+\left(\chi^{2}=20\right)$.

82 Polit $+\left(\chi^{2}=167\right)$, article $+\left(\chi^{2}=157\right)$, contemporar+ $\left(\chi^{2}=150\right)$, theor + $\left(\chi^{2}=132\right)$, concept $+\left(\chi^{2}=125\right)$, femin $+\left(\chi^{2}=123\right)$, societ $+\left(\chi^{2}=111\right)$, think $\left(\chi^{2}=106\right)$, discourse+ $\left(\chi^{2}=93\right)$, dimension $+\left(\chi^{2}=84\right)$, mainstream + $\left(\chi^{2}=84\right)$, critique+ $\left(\chi^{2}=71\right)$, approaches $\left(\chi^{2}=71\right)$.

83 Cathy J. Bradley, David Neumark, Zhehui Luo et Heather L. Bednarek, "Employment-Contingent Health Insurance, Illness, and Labor Supply of Women: Evidence from Married Women with Breast Cancer ", Health Economics, vol. 16, n 7 , juillet 2007, p. 719-737.

84 Linda J. Blumberg et al., "Toward Universal Coverage in Massachusetts ", Inquiry, vol. 43, $\mathrm{n}^{\circ}$ 2, été 2006, p. 102-121.

85 Malcolm Fraser, "Liberals and Australian Foreign Policy ", Australian Journal of Politics and History, vol. 51, $\mathrm{n}^{\circ} 3$, septembre 2005, p. 332-345. 
Classe 4 - Juridicosocial

"Transitional justice debates have generally overlooked justice, $\&$ human rights, as manifest in political, economic \& social processes whilst privileging the law, $\&$ dismissing the potential of locally generated $\&$ embedded justice ${ }^{86}$. "

Classe 5 - Politique

"The essence of the problem is that Commonwealth governments of any sort need to be seen to be active in addressing Indigenous poverty and marginalization but are unwilling to make a commitment sufficient to make a fundamental difference since there is little electoral advantage (and much electoral risk) in doing $\mathrm{so}^{87}$."

Classe 6 - Histoire

"Many scholars have historicized biopolitics with reference to the emergence of sovereign nations $\&$ their colonial extensions over the eighteenth, nineteenth \& twentieth centuries ${ }^{88}$. "

Classe 7 - Théories politicosociales

"The current literature is silent on whether democracies are more fragile or less susceptible to economic and political breakdowns. Using a number of political and policy instability variables, this article examines whether political freedom (a proxy for democracy) has any effect on the stability of political regimes $^{89}$."

\subsection{Union africaine}

Le corpus sur l'Union Africaine est peu étendu. Dans son état, il témoigne malgré tout d'une grande intrication des thématiques. Cela est tellement vrai qu'un balayage de l'ensemble des notices ne permet pas de dessiner un minimum de deux classes qui demeureraient stables. L'impossibilité, donc, de catégoriser les discours en fonction d'une statistique textuelle révèle par elle-même que les diverses questions que se posent les chercheurs ne parviennent pas à se dissocier les unes les autres.

$\overline{86}$ Paul Gready, "Reconceptualising Transitional Justice: Embedded and Distanced Justice », Conflict, Security \& Development, vol. 5, n 1, avril 2005, p. 3-21.

87 Michael Morrissey, "The Australian state and Indigenous people 19902006 ", Journal of Sociology, vol. 42, n 4, décembre 2006, p. 347-354.

88 Alison Bashford, "Global Biopolitics and the History of World Health ", History of the Human Sciences, vol. 19, $\mathrm{n}^{\circ}$ 1, février 2006, p. 67-88.

89 Abdiweli M. Ali et Hodan Said Isse, "Political Freedom and the Stability of Economic Policy ", Cato Journal, vol. 24, n 3, automne 2004, p. 251-260. 


\section{Des catégories récurrentes et peu nombreuses}

Nous avons pu mener une analyse statistique de données textuelles sur les notices des articles qui se rapportent à divers organismes internationaux. Dans six des sept cas, ces analyses sont parvenues à générer des catégories. Dans l'autre cas, les propos se sont avérés trop peu distinctifs pour que cette opération soit réalisable. Dans les six cas où une nomenclature a pu être développée, même si les analyses ont porté sur des milliers de notices, elles n'ont jamais dénombré plus de sept composants. L'hypothèse d'un nombre réduit de catégories fondamentales est donc avérée.

On pourrait nous opposer le fait que ces analyses laissent chaque fois échapper une partie du corpus; et on aurait raison de le faire. Nous pouvons néanmoins faire valoir que le corpus non appréhendé n'est jamais supérieur à $34 \%$ : il est normalement inférieur à $23 \%$. Les analyses portent donc sur l'immense majorité de l'information qui est produite par les chercheurs. Il faut bien comprendre que le discours éliminé par l'analyse ne se présente pas sous des formes récurrentes et, donc, qu'il comporte ici un caractère en partie aléatoire d'après lequel, tout au moins, il n'est pas possible de générer des catégories ou de réunir l'information.

Entre les ensembles catégoriels, il y a de nettes récurrences conceptuelles (voir Tableau 7). Il ne paraît pas possible de parler d'un organisme international sans soulever des questions économiques, politiques et sociales. Si la nomenclature est quelque peu élaborée, les formations qui en sont constitutives et qui appartiennent à un registre autre que celui de l'économie, du politique et du social côtoient sémantiquement ces trois classes prééminentes. C'est ainsi, par exemple, que la géographie se rapporte à l'économie ou au politique; que la sécurité entretient des liens de proximité avec le social et la politique; que la comptabilité est parente de l'économie; que le juridique s'approche du politique et du social; que le culturel se tient à portée du politique et du social; que l'historique met en perspective le politique et l'économique; que le théorique ou l'analytique pose un regard sur le politique, l'économique ou le social. Il semble donc difficile pour 
la communauté des chercheurs de parler d'un organisme international sans évoquer ces trois aspects essentiels que sont l'économie, le politique et le social. Si l'on dispose dans un plan factoriel, pour chaque organisme international, les catégories qui sont construites par l'analyse, on constate rapidement qu'elles ont une intersection commune, qu'elles s'enchevêtrent de telle manière qu'elles ont toujours quelque chose en partage (voir Tableau 8). Dans certains cas, elles ont aussi un univers de relative autonomie. C'est le cas de la classe qui comprend le discours social de la ZLÉA, ou de celle qui, se rapportant à l'OÉA, concerne la déviance et l'ethnicité. Mais ces univers d'autonomie relative sont généralement restreints. Les classes sont normalement interconnectées, liées entre-elles au point qu'elles semblent peu détachables les unes des autres. Ces constats expliquent sans doute pourquoi le portail de référencement en soi ne s'est pas déclaré comme réellement déterminant des contenus. Nos observations valident donc notre hypothèse de l'intrication des dimensions de la socialité ou de l'humain et confirment d'une autre manière le fait que, dans l'esprit des chercheurs, il y a des catégories fondamentales, même dans l'objet qu'est la société, c'est-à-dire au-delà même des postulats et des axiomes à partir desquels ils construisent leurs appareils analytiques.

Le discours sur les organismes internationaux est communément d'ordre social. Ce discours est souvent relié aux autres, comme en témoignent les plans factoriels. Le discours sur le social est souvent un discours éthique. Y abondent les préoccupations sur l'égalité des sexes, sur la migration, sur les droits de populations, sur les droits des travailleurs, sur le bien-être des enfants. Ces objets éthiques ne donnent pas lieu simplement à des traitements analytiques; il est courant d'y trouver des énoncés prescriptifs. Dans le corpus, les must, les should et autres have to sont fréquents, même s'il ne s'agit que de résumés des articles; on lit couramment des textes comme ceux-ci : 
Delivering the Clare Burton Memorial Lecture, Mackinnon discusses the place of young women in today's school \& society, questioning whether all girls are benefiting from the social changes that have improved the lives of some. To illustrate the contrast, she speaks first of a young European professional woman, Marian, currently working in a multinational corporation in China but planning to join her British husband in Switzerland for the birth of their first child. Where they will go next, she doesn't know, but her life will be good. Then Mackinnon focuses on a 15-year-old Australian girl, Ruby, who lives with her working-class single mother in a poor community outside Melbourne where danger \& violence are a way of life. She says she wants to become a fashion designer or hairdresser, but she is considered by her school as at risk of dropping out. Can the barriers of social class \& poverty be overcome to allow Ruby \& those like her to improve the circumstances of their lives? Can they obtain the education they need to have a career? Persistent gender inequity in school \& a labor market that cannot or does not allow women the flexibility they need to bear \& raise children are still the norm in Australia. The revolution of the 1970s \& 1980 s that sought to improve women's rights has stalled. The nation must restart it. For women to have access to a good education \& to secure, challenging employment, along with a family, is critical for them \& for Australia ${ }^{90}$.

This paper investigates the role of human capital formation in determining spatial disparities in economic performance. An informal model attempts to explain why spatial inequalities in economic performance persist over the long run. Empirical evidence indicates that the critical variable in this process is the proportion of high skill workers in a locality. Localities with a poor economic performance invariably have a low proportion of high-skill workers. The main conclusion is that government policy needs to focus on improving educational attainment and workforce skills in low income / high unemployment areas if their competitiveness is to be substantially improved $^{91}$.

In a rather unambitious working document, [4.5pc] the European Commission (2002) has presented its analysis of globalization and views on how Europe should respond to its challenges. Provoked by the blandness of this disappointing report, this paper examines whether the EU has other options than pursuing its current policies, which stay within the framework of neoliberal globalization and are

90 Alison Mackinnon, "Girls, Society and School: A Generation of Change ", Australian Feminist Studies, vol. 21, n 50, juillet 2006, p. 275-288.

91 Jim Taylor, «Spatial Inequalities in Economic Performance: The Key Role of Human Capital ", Scienze Regionali / Italian Journal of Regional Science, $\mathrm{n}^{\circ} 2$, 2003, p. 5-27. 
roughly equivalent to the ones followed and propagated by the United States $^{\text {22 }}$.

Cela confirme notre troisième hypothèse de la fréquente conjugaison du discours analytique et du discours moral.

\section{Tableau 7}

\section{Récapitulation des analyses des corpus pour chacun des organismes}

\begin{tabular}{|c|c|c|c|c|}
\hline Organismes & UDCEC* & $\begin{array}{c}\text { Nombre } \\
\text { de } \\
\text { classes }\end{array}$ & Noms des classes & $\begin{array}{l}\text { Pourcentage } \\
\text { du corpus } \\
\text { analysable }\end{array}$ \\
\hline ALÉNA & $79 \%$ & 4 & $\begin{array}{l}\text { Économie } \\
\text { Social } \\
\text { Politique } \\
\text { Géographie }\end{array}$ & $\begin{array}{l}30 \\
26 \\
22 \\
22\end{array}$ \\
\hline UE & $78 \%$ & 7 & $\begin{array}{l}\text { Économie } \\
\text { Connaissance - science } \\
\text { Politicoculturel } \\
\text { Social } \\
\text { Sécurité } \\
\text { Comptabilité } \\
\text { Politique }\end{array}$ & $\begin{array}{l}20 \\
15 \\
14 \\
14 \\
11 \\
13 \\
12\end{array}$ \\
\hline ZLÉA & $79 \%$ & 3 & $\begin{array}{l}\text { Nation - politique - philosophie } \\
\text { Analytique - économie - bien-être } \\
\text { Social }\end{array}$ & $\begin{array}{l}63 \\
27 \\
10\end{array}$ \\
\hline OÉA & $66 \%$ & 4 & $\begin{array}{l}\text { Politico-économique } \\
\text { Politicosocial } \\
\text { Analytique - déviance - ethnie } \\
\text { Social }\end{array}$ & $\begin{array}{l}16 \\
38 \\
20 \\
26\end{array}$ \\
\hline APEC & $78 \%$ & 4 & $\begin{array}{l}\text { Social } \\
\text { Géopolitique générale } \\
\text { Géopolitique spécifique } \\
\text { Économie }\end{array}$ & $\begin{array}{l}24 \\
31 \\
13 \\
32\end{array}$ \\
\hline Commonwealth & $79 \%$ & 7 & $\begin{array}{l}\text { Analyticosocial } \\
\text { Économie } \\
\text { Sécurité - géographique } \\
\text { Juridicosocial } \\
\text { Politique } \\
\text { Histoire } \\
\text { Théories politicosociales }\end{array}$ & $\begin{array}{c}20 \\
17 \\
18 \\
8 \\
8 \\
7 \\
22 \\
\end{array}$ \\
\hline Union A & N/A & N/A & N/A & N/A \\
\hline
\end{tabular}

92 Robert Went, «Globalization: Can Europe Make a Difference? », Review of International Political Economy, vol. 11, nº 5, décembre 2004, p. 980-994. 


\section{Tableau 8}

Analyse factorielle des correspondances

Représentations en coordonnées pour chacun des organismes

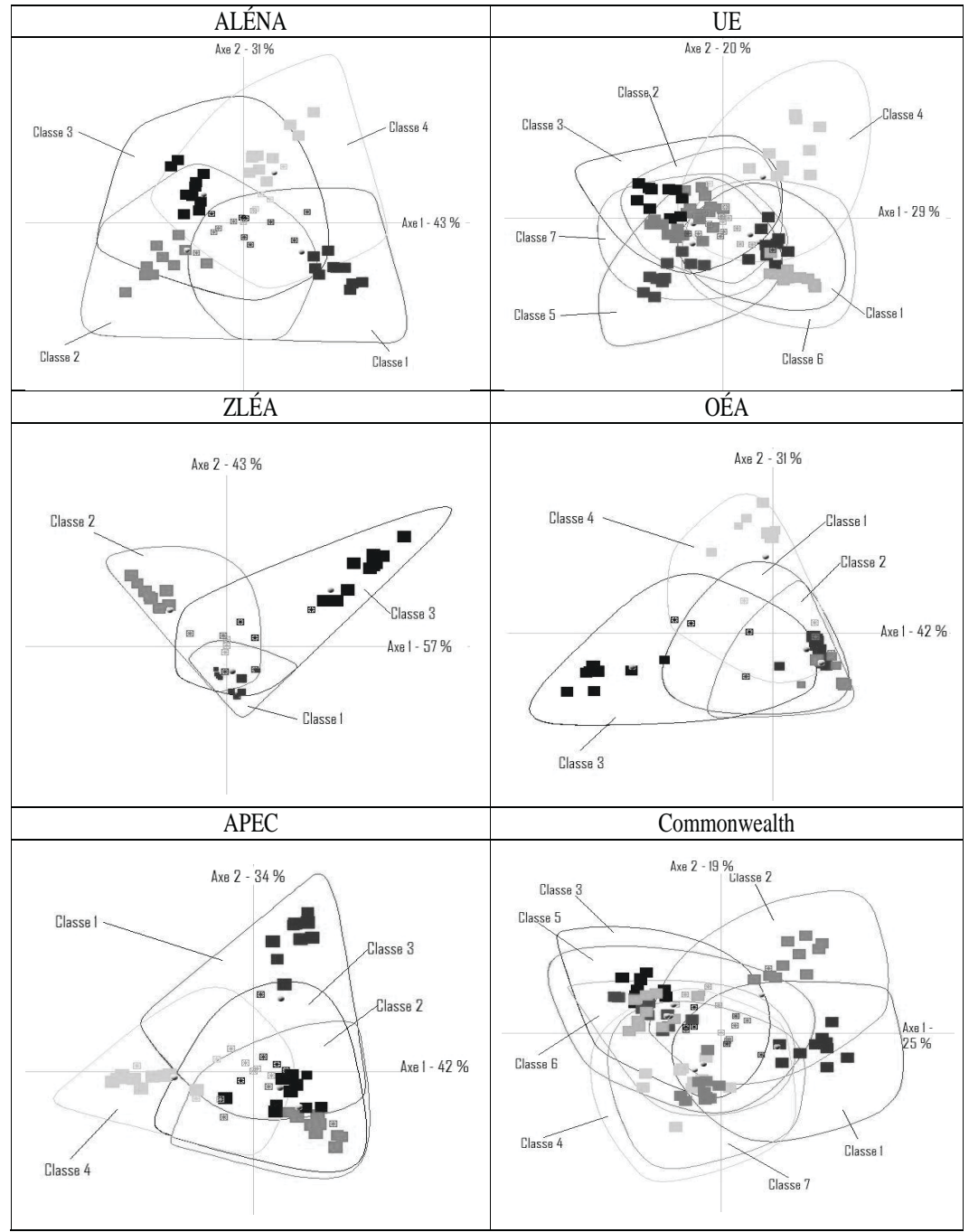




\section{Conclusion}

Il est rare que l'objet d'analyse soit constitué des travaux des chercheurs eux-mêmes; ces travaux servent normalement à fabriquer des problématiques de recherche. Il est inusité que des milliers de travaux de ce type soient pris en considération. C'est pourtant ce que rendent possible le recours aux portails de référencement et l'emploi de logiciels d'analyse de données textuelles.

Nos analyses, en se penchant sur les résumés des recherches qui ont été effectuées sur des organismes internationaux, ont découvert des domaines qui imposaient aux chercheurs leur complexité en rendant difficile la production de discours monodisciplinaires ou spécialisés, si la spécialisation doit être comprise comme intelligence d'objets réduits en leurs particules. La science du social ou de l'humain oblige les chercheurs à se côtoyer; elle les contraint même, dans la dialectique qu'ils entretiennent avec le réel, à travailler à l'intérieur de catégories générales qui minimisent les effets de la spécialisation, favorisent l'interdisciplinarité - malgré les forces de l'institution monodisciplinaire - et ouvrent sur la complexité du réel, si peu cela soit-il.

Nos résultats montrent que la spécialisation, si elle s'oriente vers l'infinitésimal, se meut également dans l'interdisciplinarité, que la compréhension des objets complexes est d'autant meilleure non pas seulement qu'elle subdivise, mais surtout qu'elle réunit. Philosophes de la synthèse et autres systémiciens ou structuralistes plaident depuis longtemps pour l'interdisciplinarité, conscients qu'ils sont de l'indissociabilité des objets d'analyse, notamment quand ils sont humains. Mais si complexe que soit le monde, il semble qu'il ne suggère pas à l'ensemble des chercheurs un nombre infini de catégories, ni sémantiques ni analytiques. Il appert que cette complexité, par elle-même, limite le nombre de catégories qui en permettent l'appréhension, comme si, la science étant ce qu'elle est à notre époque, l'indissociable pouvait être contenu dans des catégories synthétiques pourvu qu'elles se distinguent les unes des autres autant qu'elles se recoupent, comme si ces ensembles conceptuels synthétiques favorisaient la 
circulation de l'information entre les disciplines, sans rendre improbable l'originalité de propos.

Les spécialistes des sciences humaines ont communément de la difficulté à se détacher des préoccupations éthiques. Ils véhiculent même de nombreuses épistémologies où est considéré comme inadmissible ou comme impossible un propos aéthique, philosophie dont il est fort probable qu'elle soit dominante et qu' elle traverse les disciplines. Nos résultats révèlent en effet que, en sciences humaines, l'analytique et l'éthique entretiennent des relations d'intimité.

Notre analyse est peu herméneutique. On lui en fera certainement le reproche. Nous espérons que les conclusions auxquelles elle est parvenue deviendront hypothèses pour qu'une telle recherche, moins statistique, plus axée sur le sens, soit entreprise. On pourra faire aussi grief à notre analyse de n'avoir porté que sur un objet : celui des organismes internationaux. Nous croyons qu'il est, en effet, souhaitable qu'elle soit reprise sur d'autres thématiques et même sur plusieurs à la fois, afin de vérifier si nos trois conclusions (celle de la superposition des propos, celle du nombre réduit des catégories et celle du mariage de l'analytique et de l'éthique) résistent à d'autres opérationnalisations.

Mais nous retenons de notre analyse que les sciences humaines se meuvent dans l'ensemble sur un territoire d'interdisciplinarité et qu'elles appellent les modélisations où se combinent, si modestement cela soit-il, les catégories analytiques dont les propriétés sont transdisciplinaires sans pour autant prétendre à l'absoluité. 


\section{Bibliographie}

Ali, Abdiweli M. et Hodan Said Isse, "Political Freedom and the Stability of Economic Policy ", Cato Journal, vol. 24, $\mathrm{n}^{\circ}$ 3, automne 2004, p. 251-260.

Arceneaux, Craig et David Pion-Berlin, «Issues, Threats, and Institutions: Explaining OAS Responses to Democratic Dilemmas in Latin America ", Latin American Politics and Society, vol. 49, n² 2, été 2007, p. 1-31.

Barel, Yves, Le paradoxe et le système, Grenoble, Presses universitaires de Grenoble, 1989.

Bartlett, Will et Nevenka Cuckovic, "Knowledge Transfer, Institutions, and Innovation in Croatia and Slovenia ", Drustvena Istrazivanja, vol. 15, no 3, mai-juin 2006, p. 371-399.

Bashford, Alison, "Global Biopolitics and the History of World Health", History of the Human Sciences, vol. 19, n ${ }^{\circ}$ 1, février 2006, p. 67-88.

Bradley, Cathy J., David Neumark, Zhehui Luo et Heather L. Bednarek, "Employment-Contingent Health Insurance, Illness, and Labor Supply of Women: Evidence from Married Women with Breast Cancer ", Health Economics, vol. 16, n 7 , juillet 2007, p. 719-737.

Beckett, Katherine, Kris Nyrop, Lori Pfingst et Melissa Bowen, "Drug Use, Drug Possession Arrests, and the Question of Race: Lessons from Seattle ", Social Problems, vol. 52, nº 3, août 2005, p. 419-441.

Bertalanffy, Ludwig von, General System Theory: Foundations, Development, Applications, New York, George Braziller, [1968] 1976.

Blumberg, Linda J. et al., "Toward Universal Coverage in Massachusetts ", Inquiry, vol. 43, no 2, été 2006, p. 102-121.

Bourdieu, Pierre, La reproduction. Élément pour une théorie du système d'enseignement, Paris, Minuit, 1970.

Chin, Christine B. N., «Visible Bodies, Invisible Work: State Practices toward Migrant Women Domestic Workers in Malaysia ", Asian and Pacific Migration Journal, vol. 12, $\mathrm{n}^{\text {os }} 1-2,2003$, p. 49-73.

Fanning, Bryan et Fidele Mutwarasibo, "Nationals/non-nationals: Immigration, citizenship and politics in the Republic of Ireland ", Ethnic and Racial Studies, vol. 30, n ${ }^{\circ} 3$, mai 2007, p. 439-460.

Filho, Ferreira, Bento de Souza, Joaquim Mark et Jonathan Horridge, "Economic Integration, Poverty and Regional Inequality in Brazil", Revista Brasileira de Economia, vol. 60, n 4, octobre-décember 2006, p. 363-387. 
Fraser, Malcolm, "Liberals and Australian Foreign Policy ", Australian Journal of Politics and History, vol. 51, n ${ }^{\circ} 3$, septembre 2005, p. 332345.

Galasinska, Aleksandra et Dariusz Galasinski, "Shopping for a New Identity: Constructions of the Polish-German Border in a Polish Border Community ", Ethnicities, vol. 5, n 4, décembre 2005, p. 10-529.

Giddens, Antony, The Constitution of Society: Outline of the Theory of Structuration, Berkely (CA), University of California Press, 1984.

Gready, Paul, "Reconceptualising Transitional Justice: Embedded and Distanced Justice ", Conflict, Security \& Development, vol. 5, n 1 , avril 2005, p. 3-21.

Grugel, Jean, "Regionalist Governance and Transnational Collective Action in Latin America ", Economy and Society, vol. 35, n' 2, mai 2006, p. 209-231.

Haddad, Eduardo A. et Geoffrey J. D. Hewings, " Market Imperfections in a Spatial Economy: Some Experimental Results ", Quarterly Review of Economics and Finance, vol. 45, ${ }^{\text {os }} 2-3$, mai 2005, p. 476-496.

He, Baogang, "East Asian Ideas of Regionalism: A Normative Critique ", Australian Journal of International Affairs, vol. 58, $\mathrm{n}^{\circ} 1$, mai 2004, p. 105-125.

Hermann, Christoph, "Neoliberalism in the European Union ", Studies in Political Economy, n' 79 , printemps 2007, p. 61-89.

LePoire, David J., "Logistic Analysis of Recent Environmental Interest ", Technological Forecasting and Social Change, vol. 73, n 2, février 2006, p. 153-167.

Lévi-Strauss, Claude, Les structures élémentaires de la parenté, Paris, Mouton, Maison des sciences de l'homme, coll. "de Rééditions ", [1967] 1973.

Liebman, Benjamin H. et Kasaundra M. Tomlin, "Steel Safeguards and the Welfare of U.S. Steel Firms and Downstream Consumers of Steel: A Shareholder Wealth Perspective ", Canadian Journal of Economics, vol. $40, n^{\circ} 3$, août 2007 , p. $812-842$.

Lopez, Linda, "Advancing Human Rights Policy: Does Grassroots Mobilization and Community Dispute Resolution Matter? Insights from Chiapas, Mexico ", The Review of Policy Research, vol. 22, n 1 , janvier 2005, p. 77-92.

Luhmann, Niklas, Social Systems, Stanford (CA), Stanford University Press, [1984] 1995.

Mackinnon, Alison, "Girls, Society and School: A Generation of Change », Australian Feminist Studies, vol. 21, n ${ }^{\circ}$ 0, juillet 2006, p. 275-288. 
Marier, Patrik, "Affirming, Transforming, or Neglecting Gender? Conceptualizing Gender in the Pension Reform Process ", Social Politics, vol. 14, $\mathrm{n}^{\circ} 2$, été 2007, p. 182-211.

Marx, Karl, Le Capital. Critique de l'économie politique [1867], dans Euvres, Économie I, Paris, Gallimard, coll. « La pléiade », 1965.

McKay, Steven C., "The Squeaky Wheel's Dilemma: New Forms of Labor Organizing in the Philippines ", Labor Studies Journal, vol. 30, n 4 , hiver 2006, p. 41-63.

Moreau Defarges, Philippe, La mondialisation, Paris, Presses Universitaires de France, coll. "Que sais-je ?", [1997] 2005.

Morin, Edgar, La méthode - 2. La Vie de la Vie, Paris, Éditions du Seuil, 1980.

Morrissey, Michael, "The Australian state and Indigenous people 19902006 ", Journal of Sociology, vol. 42, n 4, décembre 2006, p. 347354.

Nicholls, Shelton, Anthomny Birchwood, Philip Colthrust et Earl Boodoo, "The State of and Prospects for the Deepening and Widening of Caribbean Intregration ", World Economy, vol. 23, no 9, septembre 2000, p. 1161-1194.

Nicolescu, Basarab, La transdisciplinarité. Manifeste, Éditions du Rocher, coll. "Transdisciplinarité », 1996.

Ortega, José y Gasset, "La barbarie du "spécialisme” ", dans La révolte des masses, Paris, Gallimard, coll. "Idées ", [1930] 1961.

Parsons, Talcott, The Social System, Glencoe (Ill.), Free Press, 1951.

Quanglia, Lucia, "The politics of financial services regulation and supervision reform in the European Union ", European Journal of Political Research, vol. 46, n 2 , mars 2007, p. 269-290.

Taylor, Jim, « Spatial Inequalities in Economic Performance: The Key Role of Human Capital ", Scienze Regionali / Italian Journal of Regional Science, $\mathrm{n}^{\circ}$ 2, 2003, p. 5-27.

von Bertalanffy, Ludwig, General System Theory: Foundations, Development, Applications, New York, George Braziller, [1968] 1976.

Warner, Mildred et Jennifer Gerbasi, "Rescaling and Reforming the State under NAFTA: Implications for Subnational Authority ", dans International Journal of Urban and Regional Research, vol. 28, $\mathrm{n}^{\circ} 4$, décembre 2004, p. 858-873.

Went, Robert, "Globalization: Can Europe Make a Difference? ", Review of International Political Economy, vol. 11, n 5, décembre 2004, p. $980-994$. 
Wesley, Michael, "Perspectives on Australian Foreign Policy, 2001 ", Australian Journal of International Affairs, vol. 56, $\mathrm{n}^{\circ}$ 1, avril 2002, p. 47-63.

Willett, Susan, « New Barbarians at the Gate: Losing the Liberal Peace in Africa ", Review of African Political Economy, vol. 32, n 106, décembre 2005, p. 569-594.

Wilson, John S., Catherine L. Mann et Tsunehiro Otsuki, "Trade Facilitation and Economic Development: A New Approach to Quantifying the Impact ", World Bank Economic Review, vol. 17, n 3, 2003, p. 367-389 